\title{
Evaluation of Anxiety and Depression in Caregivers of Patients Affected by Alzheimer's Disease
}

\author{
Anna Vespa ${ }^{1 *}$, Maria Velia Giulietti², Marica Ottaviani' ${ }^{3}$, R. Spatuzzi ${ }^{4}$, F. Merico ${ }^{5}$, \\ Guido Gori ${ }^{6}$, Olimpia Claudia Rossi ${ }^{3}$, L. Paciaroni ${ }^{3}$, Giuseppe Pelliccioni ${ }^{3}$, \\ Pietro Scendoni ${ }^{7}$, Cristina Meloni ${ }^{8}$ \\ ${ }^{1}$ Department of Neurology, INRCA-IRCCS Italian National Institute of Health and Science on Aging, Ancona, Italy \\ ${ }^{2}$ Unity of Neurology, INRCA-National Institute of Health and Science on Aging, Ancona, Italy \\ ${ }^{3}$ Department of Neurology, INRCA-IRCCS National Institute of Health and Science on Aging, Ancona, Italy \\ ${ }^{4}$ U.O.C. Hospice/Palliative care Departments, A.O.R. San Carlo di Potenza, Italy \\ ${ }^{5}$ Hospice Casa di Betania Palliative Care Center, Lecce, Italy \\ ${ }^{6}$ Director Day Alzheimer Center "Le Civette", ASL-Florence, Italy \\ ${ }^{7}$ Department of Rheumatology, INRCA-IRCCS National Institute of Health and Science on Aging, Fermo, Italy \\ ${ }^{8}$ INRCA-IRCCS National Institute of Health and Science on Aging, Ancona, Italy \\ Email: ${ }^{*}$ a.vespa@inrca.it, giulietti.mariavelia@libero.it, marica_O@libero.it, roberta.spatuzzi@yahoo.com, \\ fabianamerico@yahoo.it, olimpia rossi@hotmail.it, guigor@libero.it, g.pelliccioni@inrca.it, \\ p.scendoni@inrca.it, melcri86@hotmail.it
}

Received 13 January 2015; accepted 15 May 2015; published 20 May 2015

Copyright (C) 2015 by authors and Scientific Research Publishing Inc.

This work is licensed under the Creative Commons Attribution International License (CC BY).

http://creativecommons.org/licenses/by/4.0/

(c) (i) Open Access

\section{Abstract}

Background: Many studies have been underlined as care giving for people with Alzheimer's disease (AD) is highly stressful and has significant negative consequences, such as anxiety and depression. Objective: The specific aim of our study is to establish whether a difference exists in the prevalence of depression of family caregivers of Alzheimer's disease patients and healthy subjects not caregiver. Methods: Study group $(n=60)$ consists of caregivers of patients affected by Alzheimer's disease, whereas control group $(n=120)$ consists of healthy individuals who are not care giving (from at least 5 years). All the subjects were subdivided on the basis of the following independent variables: sex, age, marital status and educational level. The subjects of study and control groups studied have filled in the following tests: IPAT CDQ e IPAT ASQ Tests by Cattell which describe depression and anxiety. Such tests have been validated on the adult and elderly Italian population. Statistical analysis: Student $t$ test has been applied for the comparison between experimental and control groups. Results: The caregivers show higher levels of anxiety (medium to

\footnotetext{
${ }^{*}$ Corresponding author.
}

How to cite this paper: Vespa, A., Giulietti, M.V., Ottaviani, M., Spatuzzi, R., Merico, F., Gori, G., Rossi, O.C., Paciaroni, L., Pelliccioni, G., Scendoni, P. and Meloni, C. (2015) Evaluation of Anxiety and Depression in Caregivers of Patients Affected by Alzheimer's Disease. Advances in Alzheimer's Disease, 4, 15-20. http://dx.doi.org/10.4236/aad.2015.42003 
high, $\mathrm{P}<\mathbf{0 . 0 0 1}$ ) and depression in comparison to the group of control (medium to high, $\mathrm{P}<0.001$ ). This result indicates the caregiver shows serious depression. Conclusions: The high levels of depression and anxiety suggest the following considerations: interventions of social and psychological support are fundamental not only to maintain the patient in the family nucleus but also to maintain the caregiver's psychological health.

\section{Keywords}

\section{Caregiver, Alzheimer's Disease, Anxiety, Depression}

\section{Introduction}

The complexity of care and the constant effort make it difficult to maintain emotional balance of the caregiver and the family of a patient with Alzheimer's dementia.

The family, in fact, is still the privileged place of care for the illness of Alzheimer patient. In Italy, the caregiver is primarily a woman with a percentage up to $80 \%$ in the severe phase of illness.

The health and personal conditions of the patients and the caregivers influence the style and the quality of the care giving [1]-[4].

A progressive illness as the Alzheimer's disease leads to a profound change in the style of life of the whole family system. The changes imposed by the progression of illness set different problems in the various stadiums from a practical and organizational to the emotional point of view [3] [4]. All these can lead to the activation of new conflicts with fatigue, due to financial problems or decisions to be made. They can also reactivate ancient tensions that bring at times to definitive breakups [5]-[11].

The problematic relationship in the family system influences so much the stress of the caregiver and the quality of the daily management. The consequences invest the physical and the emotional (fatigue, scarce ability of coping) and social spheres (social isolation) [2] [5]-[17].

The deterioration of mental and physical abilities of a loved one can be for the caregiver (which has to do with behaviors related to dementia) an even bigger contributor to developing symptoms of depression. Moreover dementia- related symptoms such as wandering, agitation, hoarding and embarrassing conduct make very day challenging and make it harder for a caregiver to get rest or assistance in providing care [9] [11] [12].

In fact to assist a person with dementia can lead, therefore, to the limit the emotional resources and the caregiver can show anxious-depressive symptoms with somatic troubles that often limit the relief abilities of the caregiver [2] [5] [6] [13]-[17].

This contributes to reduce the time of permanence of the patients in the family nucleus, accelerating the appeal to live in an institution [7].

For all these reasons caregiver needs to receive psychological support [10] [12] [16]-[18].

The emotional support is a means of coping to deal with and reduce stress levels and also increases the sense of managerial competence. This promotes a caregiver emotional stability that reduces frictions and useless oppositions with positive relapses in the relationship [14] [17] [19] [20] [21].

The specific aim of our study is to establish whether a difference exists in the prevalence and depression of family caregivers of Alzheimer's disease patients and not care giving subjects. This may suggest areas of the rapeutic intervention.

\section{Methods}

Sampling-Choice of patients-The study groups was composed of $n=60$ caregivers (male and female-age range: 40 - 75 years old; care giving from 3 to 9 years) sampled randomly from a total of 170, relatives (wife, husband, son, daughter and so on) of patients affected by Alzheimer's disease. Control group $(n=104)$ consists of healthy individuals who were not care giving for almost five years (Table 1).

The caregivers were obtained from the Alzheimer Disease Daily Center of Florence, Unity of Geriatrics in Florence Hospital the Fraticini, Florence Italy. 
Table 1. Descriptive statistics of demographic variables for breast cancer patients group.

\begin{tabular}{|c|c|c|}
\hline & Caregivers $N=60$ & Healthy Subjects N =104 \\
\hline \multicolumn{3}{|l|}{ Sex } \\
\hline Male & $34 \%$ & $36 \%$ \\
\hline Female & $76 \%$ & $74 \%$ \\
\hline \multicolumn{3}{|l|}{ Age } \\
\hline Range & $40-75$ & $40-75$ \\
\hline Mean & 59.96 & 56 \\
\hline \multicolumn{3}{|l|}{ Civil Status } \\
\hline Single & $9.30 \%$ & $10.40 \%$ \\
\hline Married & $79.80 \%$ & $77.60 \%$ \\
\hline Widow & $8.50 \%$ & $7.50 \%$ \\
\hline Divorced & $2.40 \%$ & $3.50 \%$ \\
\hline \multicolumn{3}{|l|}{ Educational Level } \\
\hline Elementary School & $29.50 \%$ & $28.30 \%$ \\
\hline Middle School & $32.90 \%$ & $35.20 \%$ \\
\hline High School & $27.05 \%$ & $26 \%$ \\
\hline University & $9.30 \%$ & $10.30 \%$ \\
\hline CDQ (mean) & 6.8 & 3.7 \\
\hline
\end{tabular}

All the subjects studied were subdivided on the basis of the following independent variables sex, age marital status and educational level. All the patients were affected by Dementia of Alzheimer with clinical diagnosis through Tac.

The diagnosis of Alzheimer's disease has been carried out in accordance with the criteria of the DSM-IV while its degenerative nature has been individualized following the standardized criteria NINCDS-ADRDAAlzheimer's Criteria (specify cognitive domains) for the diagnosis of illness of Alzheimer. Particularly all the criteria have been satisfied for the diagnosis of "probable Illness of Alzheimer" (with the exception of the liquorale examination).

All the patients of this study show the level 6 of the Functional Assessment Staying Test.

The Control group was composed of $n=104$ healthy subjects chosen on the basis of the same independent variables (sex, age, marital status and educational level) of the case group. There are no differences between the two study and control groups for these variables.

Inclusion criteria included age (40 - 75 yrs). Exclusion criteria included: Refusal to participate; inability to provide informed consent; previous history of depression; use of psychotropic drugs (all, included antide- pressant).

One hundred twenty threecaregivers were approached in the clinic by the physician and asked to participate in the study. All participants signed a consensus form regarding study protocol after detailed explanation by the physician at the day center for Alzheimer disease patients. Only ninety caregivers (73.17\%) decided to par- ticipate and to fill out and sign the consent form. The caregiver was free to complete the questionnaire either in the center or at home. Caregivers electing to complete forms at home were given a self-addressed, stamped envelope to return the form.

Forty one patients have not responded to all the questions in the questionnaires: it was therefore decided not to consider them for the analysis. All subjects (case and control groups), were asked to complete the following psychological and psychosocial questionnaires:

1) Social schedule, which describes all demographic characteristics like sex, age, marital status, educational level and diagnosis, date of disease onset, disease severity, and so on.

2) IPAT-ASQ Test describing anxiety and IPAT-CDQ Test describing depression by Cattell [22] [23]. These 
tests have been used as self report methods which describe anxiety and depression respectively. The Italian version is validated on Italian population. The range is subdivided between: 0 - 3 which indicates absence or low anxiety or depression; 4 - 7 which indicates medium and medium high level of anxiety and depression; 8 - 10 which indicates high level of depression and anxiety.

\section{Statistical Analysis}

Student's t test was employed to compare study and control group. Student's t test was used to compare the variables that measure anxiety and depression.

Statistical analyses were performed with SPSS software version 17.0. P $<0.05$ was regarded as level of significance.

\section{Results}

From the comparison of Student t test between the experimental group of caregivers and the control healthy people (not care giving) a significant difference emerges for the varying anxiety $(\mathrm{P}<0.001)$.

The caregivers of patients affected by Alzheimer disease show higher (medium high) levels of anxiety in comparison to the group of control.

From the comparison of Student t test between the experimental group of caregivers and the control healthy people not assistants of family patients a significant difference emerges for the varying depression $(\mathrm{P}<0.001)$.

The caregivers show high level of depression instead of control group. This result means the caregiver shows serious depression (medium-high level CDQ) while the not care giving subjects show low levels of depression (CDQ).

The results show levels of anxiety and depression incaregiversmedium-high. These levels indicate the relevant issuesandaminor depression (Table 1).

\section{Discussion}

From the results it emerges that the family caregivers of Patients affected by Alzheimer disease have levels of anxiety and depression so high to indicate the presence of serious psychological problems.

Such result suggests some considerations.

To assist a person with dementia can bring the emotional resources to the limit and lead to anxious-depressive moods. So high level of depression, show that the caregiver may not be more able to assist the patients.

Implications for nursing care resulting from the results of this study consists in giving more support to caregivers in order to avoid their excessive involvement in caring and in guaranteeing educational interventions to help caregivers to manage the behavioral disturbances of the patients and to face on the depression and anxiety.

On the basis of these considerations it is fundamental to sustain caregivers adopting the following interventions to prevent the stress, the sense of uneasiness and the state of depression (de Rotrou, 2011, Ducharme, 2009; Ducharme 2011; Roth, 2005):

1) Psychological psychotherapy of support in sessions of group or individual.

The psychological support owes: a) give the possibility to elaborate the emotional reaction and integrate them; prevent or to face the levels of stress and to realize an acceptance of the illness (Roth, 2005).

In short, the purpose of the intervention of social and psychological support are as follows (Nápoles, 2010; de de Rotrou, 2011).

1) Facilitate management of the emotional conflicts and the stress connected to the degeneration of the personality of the patient (García-Alberca, 2012) and favour an emotional integration throw the elaboration and integration of the motional conflicts and the loss (Boots, 2013);

2) Give support from the social services.

These interventions are fundamental to maintain the psychological and physical health of the caregiver. In fact many studies have underlined that depression constitutes a factor of risk for the onset of various pathologies.

\section{Conclusions}

In conclusion this study raises a question.

The presence of high level of anxiety and depression may be markers of caregivers with a bad adaptation to 
the care giving conditions. Nurse practitioners can address depression through early detection and prevention

(Papastavrou, 2012; Epstein-Lubo, 2012).

Further studies could highlight the importance of psychological factors and personality. These factors could be used for the planning of a psychotherapeutic intervention aimed to promote the management of stress of caregivers.

There are many studies showed that the screening for caregiver burden stress can help to identify those who are at increased risk (Mohamed, 2010).

The small sample is the limitation of the present study.

\section{References}

[1] Mohamed, S., Rosenheck, R., Lyketsos, C.G. and Schneider, L.S. (2010) Caregiver Burden in Alzheimer Disease: Cross-Sectional and Longitudinal Patient Correlates. The American Journal of Geriatric Psychiatry, 18, 917-927. http://dx.doi.org/10.1097/JGP.0b013e3181d5745d

[2] Schulz, R., McGinnis, K.A., Zhang, S., Martire, L.M., Hebert, R.S., Beach, Scott, R., et al. (2008) Dementia Patient Suffering and Caregiver Depression. Alzheimer Disease \& Associated Disorders, 22, 170-176. http://dx.doi.org/10.1097/WAD.0b013e31816653cc

[3] Mausbach, B.T., Chattillion, E., Roepke, S.K., Ziegler, M.G., Milic, M., von Känel, R., et al. (2012) A Longitudinal Analysis of the Relations among Stress, Depressive Symptoms, Leisure Satisfaction, and Endothelial Function in Caregivers. Health Psychology, 31, 433-440. http://dx.doi.org/10.1037/a0027783

[4] García-Alberca, J.M., Cruz, B., Lara, J.P., Garrido, V., Lara, A., Gris, E. and Gonzalez-Herero, V. (2013) The Experience of Caregiving: The Influence of Coping Strategies on Behavioral and Psychological Symptoms in Patients with Alzheimer's Disease. Aging \& Mental Health, 17, 615-622. http://dx.doi.org/10.1080/13607863.2013.765833

[5] Papastavrou, E., Charalambous, A., Tsangari, H. and Karayiannis, G. (2012) The Burdensome and Depressive Experience of Caring: What Cancer, schizopHrenia, and Alzheimer's Disease Caregivers Have in Common. Cancer Nursing, 35, 187-194. http://dx.doi.org/10.1097/NCC.0b013e31822cb4a0

[6] Papastavrou, E., Kalokerinou, A., Papacostas, S.S., Tsangari, H. and Sourtzi, P. (2007) Caring for a Relative with Dementia: Family Caregiver Burden. Journal of Advanced Nursing, 58, 446-457. http://dx.doi.org/10.1111/j.1365-2648.2007.04250.x

[7] Epstein-Lubo, G., Gaudiano, B., Darling, E., Hinckley, M., Tremont, G., Kohn, R., et al. (2012) Differences in Depression Severity in Family Caregivers of Hospitalized Individuals with Dementia and Family Caregivers of Outpatients with Dementia. The American Journal of Geriatric Psychiatry, 20, 815-819. http://dx.doi.org/10.1097/JGP.0b013e318235b62f

[8] Roth, D.L., Mittelman, M.S., Clay, O.J., Madan, A. and Haley, W.E. (2005) Changes in Social Supports as Mediators of the Impact of a Psychosocial Intervention for Spouse Caregivers of Persons with Alzheimer's Disease. Psychology and Aging, 20, 634- 644. http://dx.doi.org/10.1037/0882-7974.20.4.634

[9] Papastavrou, E., Tsangari, H., Karayiannis, G., Papacostas, S., Efstathiou, G. and Sourtzi, P. (2011) Caring and Coping: The Dementia Caregivers. Aging \& MentL Health, 15, 702-711. http://dx.doi.org/10.1080/13607863.2011.562178

[10] García-Alberca, J.M., Cruz, B., Lara, J.P., Garrido, V., Gris, E., Lara, A. and Castilla, C. (2012) Disengagement Coping Partially Mediates the Relationship between Caregiver Burden and Anxiety and Depression in Caregivers of People with Alzheimer’s Disease. Results from the MÁLAGA-AD Study. Journal of Affective Disorders, 136, 848-856. http://dx.doi.org/10.1016/j.jad.2011.09.026

[11] Watson, L.C., Lewis, C.L., Moore, C.G. and Jeste, D.V. (2011) Perceptions of Depression among Dementia Caregivers: Findings from the CATIE-AD Trial. International Journal of Geriatric Psychiatry, 26, 397-402. http://dx.doi.org/10.1002/gps.2539

[12] Rocca, P., Leotta, D., Liffredo, C., Mingrone, C., Sigaudo, M., Capellero, B., et al. (2010) Neuropsychiatric Symptoms Underlying Caregiver Stress and Insight in Alzheimer's Disease. Dementia and Geriatric Cognitive Disorders, 30, 57-63. http://dx.doi.org/10.1159/000315513

[13] Berger, G., Bernhardt, T., Weimer, E., Peters, J., Kratzsch, T. and Frolich, L. (2005) Longitudinal Study on the Relationship between Symptomatology of Dementia and Levels of Subjective Burden and Depression among Family Caregivers in Memory Clinic Patients. Journal of Geriatric Psychiatry and Neurology, 18, 119-128. http://dx.doi.org/10.1177/0891988704273375

[14] Thomas, P., Lalloué, F., Preux, P.M., Hazif-Thomas, C., Pariel, S., Inscale, P., et al. (2006) Dementia Patients Caregivers Quality of Life: The PIXEL Study. International Journal of Geriatric Psychiatry, 21, 50-56. http://dx.doi.org/10.1002/gps.1422 
[15] Burgio, L., Stevens, A., Guy, D., Roth, D.L. and Haley, W.E. (2003) Impact of Two Psychosocial Interventions on White and African American Family Caregivers of Individuals with Dementia. The Gerontologist, 43, 568-579. http://dx.doi.org/10.1093/geront/43.4.568

[16] Boots, L.M., de Vugt, M.E., van Knippenberg, R.J., Kempen, G.I.J.M. and Verhey, F.R.J. (2013) A Systematic Review of Internet-Based Supportive Interventions for Caregivers of Patients with Dementia. International Journal of Geriatric Psychiatry, 29, 331-334. http://dx.doi.org/10.1002/gps.4016

[17] García-Alberca, J.M., Cruz, B., Lara, J.P., Garrido, V., Lara, A. and Gris, E. (2012) Anxiety and Depression Are Associated with Coping Strategies in Caregivers of Alzheimer’s Disease Patients: Results from the MÁLAGA-AD Study. International Psychogeriatrics, 24, 1325-1334. http://dx.doi.org/10.1017/S1041610211002948

[18] de Rotrou, J., Cantegreil, I., Faucounau, V., Wenisch, E., Chausson, C., Jegou, D., et al. (2011) Do Patients Diagnosed with Alzheimer's Disease Benefit from a Psycho-Educational Programme for Family Caregivers? A Randomised Controlled Study. International Journal of Geriatric Psychiatry, 26, 833-842. http://dx.doi.org/10.1002/gps.2611

[19] Nápoles, A.M., Chadiha, L., Eversley, R. and Moreno-John, G. (2010) Developing Culturally Sensitive Dementia Caregiver Interventions: Are We There Yet? American Journal of Alzheimer's Disease and Other Dementias, 25, 389406. http://dx.doi.org/10.1177/1533317510370957

[20] Ducharme, F.C., Lévesque, L.L., Lachance, L.M., Kergoat, M.-J., Legault, A.J., Beaudet, L.M. and Zarit, S.H. (2011) "Learning to Become a Family Caregiver" Efficacy of an Intervention Program for Caregivers Following Diagnosis of Dementia in a Relative. The Gerontologist, 51, 484- 494. http://dx.doi.org/10.1093/geront/gnr014

[21] Ducharme, F., Beaudet, L., Legault, A., Kergoat, M.-J., Lévesque, L. and Caron, C. (2009) Development of an Intervention Program for Alzheimer’s Family Caregivers Following Diagnostic Disclosure. Clinical Nursing Research, 18, 44-67. http://dx.doi.org/10.1177/1054773808330093

[22] Vigneau, F. and Cormier, S. (2008) The Factor Structure of the State-Trait Anxiety Inventory: An Alternative View. Journal of Personality Assessment, 90, 280-285. http://dx.doi.org/10.1080/00223890701885027

[23] Ramanaiah, N.V., Franzen, M. and Schill, T. (1983) A Psychometric Study of the State-Trait Anxiety Inventory. Journal of Personality Assessment, 47, 531-535. http://dx.doi.org/10.1207/s15327752jpa4705 14 